\title{
The Role of Vitamin D in Non-Scarring Alopecia
}

\author{
Agnieszka Gerkowicz *, Katarzyna Chyl-Surdacka, Dorota Krasowska and \\ Grażyna Chodorowska
}

Chair and Department of Dermatology, Venereology and Paediatric Dermatology, Medical University of Lublin, Radziwiłłowska 13, 20-080 Lublin, Poland; kasiachyl@gmail.com (K.C.-S.); dorota.krasowska@umlub.pl (D.K.); grazyna.chodorowska@umlub.pl (G.C.)

* Correspondence: agerkowicz@wp.pl; Tel.: +48-81-532-36-47

Received: 29 October 2017; Accepted: 3 December 2017; Published: 7 December 2017

\begin{abstract}
Non-scarring hair loss is a common problem that affects both male and female patients. Since any disturbances in the hair follicle cycle may lead to hair shedding, or alopecia, it is not surprising that the possible role of vitamin D in alopecia was investigated in many studies. Vitamin $\mathrm{D}$ has been shown to have many important functions. A growing body of evidence shows that vitamin $\mathrm{D}$ and its receptor are responsible for maintaining not only calcium homeostasis but also skin homeostasis. Moreover, vitamin D could also regulate cutaneous innate and adaptive immunity. This paper presents a review of current literature considering the role of vitamin D in alopecia areata, telogen effluvium, and female pattern hair loss. The majority of studies revealed decreased serum 25-hydroxyvitamin D levels in patients with different types of non-scarring alopecia, which could suggest its potential role in the pathogenesis of hair loss. According to the authors, vitamin D supplementation could be a therapeutic option for patients with alopecia areata, female pattern hair loss, or telogen effluvium. However, further studies on a larger group of patients are required.
\end{abstract}

Keywords: vitamin D; vitamin D receptor; alopecia

\section{Introduction}

Vitamin D plays an important role in human health. Its main source is photosynthesis in the skin, whereas lower amounts are derived from nutrition and diet supplements [1]. The role of vitamin $\mathrm{D}$ in regulation of calcium homeostasis is well established. It stimulates the intestinal absorption of calcium and phosphate, reduces their renal clearance, and promotes bone mineralization [2-4]. Apart from calcium homeostasis, vitamin D has also been shown to modulate both innate and adaptive immunity [5]. It stimulates differentiation of monocytes into classical macrophages and enhances the chemotactic and phagocytic capacity of macrophages [6]. Wang et al. [7] demonstrated that 1,25-dihydroxyvitamin $\mathrm{D}\left(1,25(\mathrm{OH})_{2} \mathrm{D}\right)$ activates the transcription of antimicrobial peptides such as cathelicidin antimicrobial peptide in isolated human keratinocytes, monocytes, and neutrophils and enhances expression of defensin $\beta 2$ in primary cultures of adult keratinocytes. Moreover, they observed that $1,25(\mathrm{OH})_{2} \mathrm{D}$ alone or in conjunction with lipopolysaccharide (LPS) induced cathelicidin antimicrobial peptide expression and release of antimicrobial activity in neutrophils. It has been proposed that induction of antimicrobial peptide expression by $1,25(\mathrm{OH})_{2} \mathrm{D}$ could be involved in the suppressive effects of ultraviolet $B(\mathrm{UVB})$ radiation on innate immunity and suggested the potential of its analogues in treatment of opportunistic infections [7]. Furthermore, $1,25(\mathrm{OH})_{2} \mathrm{D}$ has been found to suppress antigen presentation by dendritic cells [8] and to modulate the function of Thelper (Th) lymphocytes by suppressing the synthesis of T helper type 1 (Th1) proinflammatory cytokines and enhancing cytokine production by T helper type 2 (Th2) cells [9]. 1,25(OH) 2 D has also been shown to suppress the $\mathrm{T}$ helper type 17 (Th17) related cytokine expression [10]. Besides Th lymphocytes, 
$1,25(\mathrm{OH})_{2} \mathrm{D}$ also induces apoptosis in activated B cells, suppresses B-cell proliferation, and decreases plasma cell and class-switched memory B-cell generation [11].

Since the immunomodulatory effect of vitamin $\mathrm{D}$ was demonstrated, its potential role has been studied in many areas of medicine. A growing body of evidence shows that vitamin D and its receptor may be involved in skin homeostasis [1,4,12-16].

Skin is a target organ for vitamin D not only because this compound is mostly synthesized in the epidermal keratinocytes and dermal fibroblasts from 7-dehydrocholesterol upon exposure to UVB radiation, but also because skin is able to respond to the active metabolites of vitamin D [1]. As mentioned above, vitamin D synthesis begins in the skin. The previtamin D is subsequently converted by a spontaneous thermal isomerization into vitamin $\mathrm{D}$, which after bonding to a carrier protein is transported to the liver and hydroxylated to 25-hydroxyvitamin $\mathrm{D}(25(\mathrm{OH}) \mathrm{D})$. The serum level of this form is a marker of vitamin D status. However, the biologically active form 1,25-dihydroxyvitamin $\mathrm{D}\left(1,25(\mathrm{OH})_{2} \mathrm{D}\right)$ is formed in the kidneys [1].

It was demonstrated that keratinocytes are capable of metabolizing vitamin $\mathrm{D}$ to the active form because they possess the activity of 1- $\alpha$-hydroxylase and 25-hydroxylase [17]. Other cells including macrophages and dendritic cells are also capable of synthesizing the active form of vitamin $\mathrm{D}$, and this process is predominantly regulated by immune signals, but not by calcium or parathyroid hormone concentration [18]. This is important because many of those cells are involved in the pathogenesis of different skin diseases $[12,18,19]$.

Vitamin D exerts its action through the vitamin D receptor (VDR) [4,18-21]. VDR is expressed in keratinocytes, dendritic cells, macrophages, $\mathrm{B}$ and $\mathrm{T}$ lymphocytes, and in two major cell populations in the hair follicle: epidermal keratinocytes and mesodermal dermal papilla cells $[12,19,22]$. VDR is crucial for hair follicle integrity [22]. Its expression is required for normal hair follicle cycling but not for morphogenesis, and its deficiency can inhibit keratinocytes differentiation and disturb the normal postnatal hair follicle cycle $[12,17,19,23]$. It was demonstrated that patients with type IIA vitamin D-dependent rickets (VDDR IIA) develop alopecia between 1-3 months of age together with osteomalacia, dental caries, hyperparathyroidism, and mineral disturbances, including hypocalcaemia and hypophosphatemia [23-25]. Histopatological studies of patients with VDDR IIA demonstrated abnormalities of the hair follicles, including the presence of dermal cysts and irregular epidermal structures in the lower part of the hair follicle [25]. It was demonstrated that in VDR null mice, the hair follicles in catagen become dystrophic and the dermal papilla separates from the rest of the hair follicle as catagen progresses; consequently, anagen is not reinitiated [26]. Therefore, it has been proposed that VDR is required for anagen initiation [1]. Histologic changes within the hair follicle similar to those described in VDDR IIA were observed in atrichia with papular lesions (APL), characterized by total alopecia and papular and milia like growths that developed after birth [25]. Hence, it has been suggested that the VDR gene and Hairless gene ( $\mathrm{Hr} r$ may be involved in the same genetic pathway that regulates postnatal hair cycling $[23,25,27]$. Moreover, it was found that VDR deficiency leads to increased $\mathrm{Hr}$ expression [26].

Since the role of VDR in the hair follicle cycle was confirmed, the potential role of vitamin D was the focus of many studies. It has been demonstrated that VDR knockout mice have increased levels of the active form of vitamin D. However, the possible toxic effect of 1,25-dihydroxyvitamin D on hair follicles was not proven [28]. Recently, Mady et al. demonstrated a possible role of calcium and $1,25(\mathrm{OH})_{2} \mathrm{D}$ in the regulation of postnatal hair follicle cycling [29]. They found that calbidin- $\mathrm{D}_{9 \mathrm{k}}$ knockout pups born of calbidin- $\mathrm{D}_{9 \mathrm{k}}$ knockout females fed with a low-calcium and vitamin D-deficient diet developed transient alopecia during the first postnatal catagen. However, alopecia was not present when these pups were suckled by mothers fed with a diet containing calcium and vitamin D [29].

Considering that any disturbances in the hair follicle cycle may lead to hair loss, it is not surprising that the possible role of vitamin D in alopecia was investigated in many studies. The aim of this paper is to review the current literature considering the role of vitamin D in non-scarring alopecia. 


\section{Vitamin D in Alopecia Areata}

Alopecia areata (AA) is a common form of hair loss, characterized by sharply demarcated, round to oval, skin-coloured patches of non-scarring alopecia [30,31]. Study results support the autoimmune nature of AA, including: association with other autoimmune diseases, presence of hair follicle-specific autoantibodies, or improvement after immunosuppressive treatment [30-32]. Typical histological features of AA are: inflammatory infiltrate around and within hair follicles, composed mainly of Th1 cells and increased expression of interleukin-2 (IL-2) and interferon $\gamma$ (IFN $\gamma$ ) [30-32]. At present, studies concentrate on finding the mediators involved in the pathogenic processes in AA, which could be a target for new therapeutic options [30,31].

Literature data suggest that vitamin $\mathrm{D}$, due to its immunomodulatory effect, may be involved in the pathogenesis of AA [12,16,33].

Decreased serum 25(OH)D levels in AA in comparison to healthy subjects were reported in many studies [12,13,16,33-37] (Table 1).

Mahamid et al. suggested that serum $25(\mathrm{OH}) \mathrm{D}$ levels $<30 \mathrm{ng} / \mathrm{mL}$ were associated with AA occurrence [16]. D'Ovidio et al. reported that decreased serum $25(\mathrm{OH}) \mathrm{D}$ levels were accompanied by a compensatory increase of parathyroid hormone levels, which confirmed the real deficiency of vitamin $\mathrm{D}$ in patients with AA [13].

Interestingly, data concerning the correlation between serum 25(OH)D levels and clinical disease parameters are inconsistent. Yilmaz et al. did not find any correlation between $1,25(\mathrm{OH})_{2} \mathrm{D}$ or $25(\mathrm{OH}) \mathrm{D}$ concentrations in 42 patients with AA and the extent of hair loss, number of patches, disease duration, and nail involvement [33]. This was confirmed in another study [36]. In contrast, Aksu Cerman et al. demonstrated for the first time that serum 25(OH)D levels were inversely correlated with severity of AA assessed by Severity of Alopecia Tool (SALT) score [12]. This correlation was observed in further studies $[34,35,37]$. It has been suggested that lower serum levels of $25(\mathrm{OH}) \mathrm{D}$ found in more severe stages of AA might result from emotional stress due to severe hair loss which might discourage patients from public appearance and therefore from sun exposure [12]. However, it cannot be excluded that the observed differences between studies might result from different enrolment criteria, including AA severity and an unequal number of examined patients [13,33,35-37]. Moreover, another possible explanation could be the seasonal variation in serum 25(OH)D levels. Aksu Cerman et al. conducted a study during winter, whereas Yilmaz et al. during summer [12,33]. In addition, data regarding serum vitamin D levels among female and male patients with AA are also inconclusive. There are studies that point to lower levels in male patients than in females [13,36], studies that present opposite results [12,37] and studies that demonstrate no correlation with gender [34]. This can be explained by different cultural and religious aspects that influence vitamin D synthesis $[12,33,34,36]$. 
Table 1. Clinical studies evaluating serum concentration of 25(OH)D in non-scarring alopecia.

\begin{tabular}{|c|c|c|c|c|c|}
\hline \multirow[b]{2}{*}{ Authors } & \multirow{2}{*}{$\begin{array}{l}\text { Study Subjects (Number } \\
\text { and Age) }\end{array}$} & \multirow{2}{*}{ Severity of Alopecia } & \multicolumn{3}{|c|}{ Serum Concentration of Vitamin D $(25(\mathrm{OH}) \mathrm{D})$} \\
\hline & & & $\begin{array}{l}\text { Criteria for Vitamin D } \\
\text { Status }\end{array}$ & $\begin{array}{l}\text { Method of Serum } \\
\text { 25(OH)D Measurement }\end{array}$ & Notable Findings \\
\hline $\begin{array}{l}\text { Aksu Cerman } \\
\text { et al., } 2014 \text { [12] }\end{array}$ & $\begin{array}{l}\text { AA-86 patients } \\
\text { V-44 patients } \\
\text { C-58 healthy controls } \\
\text { Mean age of: } \\
\text { AA-32.21 } \pm 9.60 \text { years } \\
\text { V-33.64 } \pm 11.51 \text { years } \\
\text { C-32.55 } \pm 9.78 \text { years }\end{array}$ & $\begin{array}{l}\text { S1-71 patients } \\
\text { S2-15 patients }\end{array}$ & $\mathrm{D} \leq 20 \mathrm{ng} / \mathrm{mL}^{-1}$ & LC-MS/MS & $\begin{array}{l}\text { Significantly higher prevalence of } \\
\text { vitamin } \mathrm{D} \text { deficiency in AA than in } \\
\mathrm{V} \text { and } \mathrm{C}(p=0.003, p<0.001 \\
\text { respectively). } \\
\text { A significant negative correlation } \\
\text { between AA severity and serum } \\
\text { concentration of } 25(\mathrm{OH}) \mathrm{D} \\
(p<0.001 r=-0.409) .\end{array}$ \\
\hline $\begin{array}{l}\text { D'Ovidio R et } \\
\text { al., } 2013 \text { [13] }\end{array}$ & $\begin{array}{l}\text { AA- } 156 \text { patients } \\
\text { C-148 healthy controls } \\
\text { Mean age of: } \\
\text { AA-37.8 years } \\
\text { C-34.5 years }\end{array}$ & $\begin{array}{l}\text { AA multilocularis-49 } \\
\text { patients } \\
\text { Ophiasis-69 patients } \\
\text { AT-AU—38 patients } \\
\text { Minimal hair loss in all } \\
\text { groups: } 25 \% \text { of scalp area }\end{array}$ & $\mathrm{D}<20 \mathrm{ng} / \mathrm{mL}$ & CHL & $\begin{array}{l}\text { Presence of serum } 25(\mathrm{OH}) \mathrm{D} \text { levels } \\
<20 \mathrm{ng} / \mathrm{mL} \text { significantly higher in } \\
\text { AA vs. C }(p<0.025) \text {. } \\
\text { In AA higher compensatory levels } \\
\text { of PTH }(r=-0.24, p<0.01) \text {; }\end{array}$ \\
\hline $\begin{array}{l}\text { Mahamid et al., } \\
2014 \text { [16] }\end{array}$ & $\begin{array}{l}\text { AA }-23 \text { patients } \\
\text { C }-20 \text { healthy controls } \\
\text { Mean age of: } \\
\text { AA }-24.2 \pm 12.3 \text { years } \\
C-27 \pm 11.26 \text { years }\end{array}$ & $\begin{array}{l}\text { Patchy AA-18 } \\
\text { Extensive AA-5 }\end{array}$ & $\begin{array}{l}\mathrm{S}-30-50 \mathrm{ng} / \mathrm{mL} \\
\mathrm{I}<30 \mathrm{ng} / \mathrm{mL} \\
\mathrm{D}<20 \mathrm{ng} / \mathrm{mL}\end{array}$ & EIA & $\begin{array}{l}\text { Serum } 25(\mathrm{OH}) \mathrm{D} \text { concentration } \\
\text { significantly decreased in AA vs. C } \\
(p<0.05) ; \\
\text { Serum } 25(\mathrm{OH}) \mathrm{D} \text { levels }<30 \mathrm{ng} / \mathrm{mL} \\
\text { and CRP }>1 \text { associated with AA } \\
\text { occurrence }(p=0.02, p=0.04 \\
\text { respectively). }\end{array}$ \\
\hline $\begin{array}{l}\text { Yilmaz et al., } \\
2012 \text { [33] }\end{array}$ & $\begin{array}{l}\text { AA }-42 \text { patients } \\
\text { C }-42 \text { healthy controls } \\
\text { Mean age of: } \\
\text { AA-30.8 } \pm 8.2 \text { years } \\
\text { C }-29.3 \pm 7.4 \text { years }\end{array}$ & $\begin{array}{l}\text { S1-30 patients } \\
\text { S2-6 patients } \\
\text { S3-3 patients } \\
\text { S4-2 patients } \\
\text { S5-1 patient }\end{array}$ & $\begin{array}{l}25(\mathrm{OH}) \mathrm{D} \text {-insufficient } \\
\text { concentration }< \\
50 \mathrm{nmol} / \mathrm{L} \\
1,25(\mathrm{OH})_{2} \mathrm{D} \text {-decreased } \\
\text { concentrations } \leq \\
30 \mathrm{pg} / \mathrm{mL}\end{array}$ & ELISA & $\begin{array}{l}\text { Significantly lower concentration of } \\
25(\mathrm{OH}) \mathrm{D} \text { and } 1,25(\mathrm{OH})_{2} \mathrm{D} \text { in AA vs } \\
\mathrm{C}(p<0.001, p<0.001 \text { respectively) } \\
\text { No correlation between the levels } \\
\text { of } 25(\mathrm{OH}) \mathrm{D} \text { and } 1,25(\mathrm{OH})_{2} \mathrm{D} \text { and } \\
\text { disease severity, duration, nail } \\
\text { involvement. }\end{array}$ \\
\hline $\begin{array}{l}\text { Bakry et al., } \\
2016 \text { [34] }\end{array}$ & $\begin{array}{l}\text { AA-60 patients } \\
\text { C-60 healthy controls } \\
\text { Mean age of: } \\
\text { AA-20.70 } \pm 10.85 \text { years } \\
C-23.71 \pm 7.45 \text { years }\end{array}$ & $\begin{array}{l}\text { Mild-24 patients } \\
\text { Moderate-20 patients } \\
\text { Severe-16 patients }\end{array}$ & $\begin{array}{l}\mathrm{S}>75 \mathrm{nmol} / \mathrm{L} \\
\mathrm{I}-50-75 \mathrm{nmol} / \mathrm{L} \\
\mathrm{D}<50 \mathrm{nmol} / \mathrm{L}\end{array}$ & ELISA & $\begin{array}{l}\text { Significantly lower levels of serum } \\
25(\mathrm{OH}) \mathrm{D} \text { in AA vs. C }(p<0.001) \text {. } \\
\text { Significantly lower serum levels of } \\
25(\mathrm{OH}) \mathrm{D} \text { in severe AA vs. } \\
\text { moderate and mild }(p=0.03, \\
p=0.002 \text { respectively). }\end{array}$ \\
\hline
\end{tabular}


Table 1. Cont.

\begin{tabular}{|c|c|c|c|c|c|}
\hline \multirow[b]{2}{*}{ Authors } & \multirow{2}{*}{$\begin{array}{l}\text { Study Subjects (Number } \\
\text { and Age) }\end{array}$} & \multirow[b]{2}{*}{ Severity of Alopecia } & \multicolumn{3}{|c|}{ Serum Concentration of Vitamin D $(25(\mathrm{OH}) \mathrm{D})$} \\
\hline & & & $\begin{array}{l}\text { Criteria for Vitamin D } \\
\text { Status }\end{array}$ & $\begin{array}{l}\text { Method of Serum } \\
\text { 25(OH)D Measurement }\end{array}$ & Notable Findings \\
\hline $\begin{array}{l}\text { Ghafoor et al., } \\
2017 \text { [35] }\end{array}$ & $\begin{array}{l}\text { AA-30 patients } \\
\text { C-30 healthy controls } \\
\text { Mean age of: } \\
\text { AA }-23.77 \pm 8.86 \text { years } \\
C-24.03 \pm 8.62 \text { years }\end{array}$ & $\begin{array}{l}\text { S1-4 patients } \\
\text { S2-7 patients } \\
\text { S3-12 patients } \\
\text { S4-1 patient } \\
\text { S5-6 patients }\end{array}$ & $\begin{array}{l}\mathrm{S}-30 \mathrm{ng} / \mathrm{dL} \\
\mathrm{I}-20-29 \mathrm{ng} / \mathrm{dL} \\
\mathrm{D}<20 \mathrm{ng} / \mathrm{dL}\end{array}$ & EIA & $\begin{array}{l}\text { Significantly lower serum } 25(\mathrm{OH}) \mathrm{D} \\
\text { levels in AA vs. C }(p=0.001) \text {. } \\
\text { Lower serum } 25(\mathrm{OH}) \mathrm{D} \text { levels in } \\
\text { patients with higher SALT Score. }\end{array}$ \\
\hline $\begin{array}{l}\text { Darwish et al., } \\
2017 \text { [36] }\end{array}$ & $\begin{array}{l}\text { AA-30 patients } \\
\text { C-20 healthy controls } \\
\text { Mean age of: } \\
\text { AA-28.67 } \pm 10 \text { years } \\
\text { C }-24.8 \pm 6 \text { years }\end{array}$ & $\begin{array}{l}\text { S1 (mild) - } 10 \text { patients } \\
\text { S2 (moderate)-7 patients } \\
\text { S3-S5 (severe)-13 } \\
\text { patients }\end{array}$ & NA & ELISA & $\begin{array}{l}\text { Significant decrease of serum } \\
25(\mathrm{OH}) \mathrm{D} \text { concentration in AA vs. C } \\
(p<0.001) . \\
\text { In AA significantly lower serum } \\
\text { 25(OH)D level in males vs. females } \\
(p=0.009) \text {. } \\
\text { No correlation with SALT score. }\end{array}$ \\
\hline $\begin{array}{l}\text { Attawa et al., } \\
2016[37]\end{array}$ & $\begin{array}{l}\text { AA-23 patients } \\
\text { C-23 healthy controls } \\
\text { Mean age of: } \\
\text { AA-26.44 } \pm 10.87 \text { years } \\
C-29.39 \pm 8.10 \text { years }\end{array}$ & $\begin{array}{l}\text { S1-14 patients } \\
\text { S2-3 patients } \\
\text { S3-S5-6 patients }\end{array}$ & $\begin{array}{l}\mathrm{S}>30 \mathrm{ng} / \mathrm{mL} \\
\mathrm{I}-10-30 \mathrm{ng} / \mathrm{mL} \\
\mathrm{D}<10 \mathrm{ng} / \mathrm{mL}\end{array}$ & ELISA & $\begin{array}{l}\text { Significantly lower serum } 25(\mathrm{OH}) \mathrm{D} \\
\text { levels in AA vs. C }(p=0.01) \text {. } \\
\text { Significant difference between } \\
\text { vitamin D status and AA severity } \\
(p=0.02) .\end{array}$ \\
\hline $\begin{array}{l}\text { Erpolat et al., } \\
2017 \text { [38] }\end{array}$ & $\begin{array}{l}\text { AA-41 patients } \\
\text { C-32 healthy controls } \\
\text { Mean age of: } \\
\text { AA cases-32.8 } \pm 7.5 \\
\text { years } \\
\text { C-32.7 } \pm 7.5 \text { years }\end{array}$ & $\begin{array}{l}\text { Single patch-15 patients } \\
\text { Multiple patches- } 26 \\
\text { patients }\end{array}$ & $\begin{array}{l}\mathrm{S}>30 \mathrm{ng} / \mathrm{mL} \\
\mathrm{I}-20-30 \mathrm{ng} / \mathrm{mL} \\
\mathrm{D}<20 \mathrm{ng} / \mathrm{mL}\end{array}$ & HPLC & $\begin{array}{l}\text { No significant difference in serum } \\
25(\mathrm{OH}) \mathrm{D} \text { levels between AA and } \\
\text { control }(p>0.05) \text {. } \\
\text { Vitamin D deficiency-93.8\% } \\
\text { patients with AA }\end{array}$ \\
\hline $\begin{array}{l}\text { Bhat et al., } \\
2017 \text { [39] }\end{array}$ & $\begin{array}{l}\text { AA-50 patients } \\
\text { C-35 healthy controls } \\
\text { Mean age of: } \\
\text { AA cases-22.4 } \pm 8.6 \\
\text { years } \\
\text { C-29.2 } \pm 7.6 \text { years }\end{array}$ & $\begin{array}{l}\text { S1-38 patients } \\
\text { S2*-12 patients }\end{array}$ & $\mathrm{D}<30 \mathrm{ng} / \mathrm{mL}$ & CHL & $\begin{array}{l}\text { Serum } 25(\mathrm{OH}) \mathrm{D} \text { levels significantly } \\
\text { lower in AA vs. C }(p<0.001) \text {. } \\
\text { A significant negative correlation } \\
\text { between SALT score and serum } \\
\text { vitamin D levels }(p<0.001 \text {; } \\
r=-0.730) \text {. }\end{array}$ \\
\hline
\end{tabular}


Table 1. Cont.

\begin{tabular}{|c|c|c|c|c|c|}
\hline \multirow[b]{2}{*}{ Authors } & \multirow{2}{*}{$\begin{array}{l}\text { Study Subjects (Number } \\
\text { and Age) }\end{array}$} & \multirow{2}{*}{ Severity of Alopecia } & \multicolumn{3}{|c|}{ Serum Concentration of Vitamin D $(25(\mathrm{OH}) \mathrm{D})$} \\
\hline & & & $\begin{array}{l}\text { Criteria for Vitamin D } \\
\text { Status }\end{array}$ & $\begin{array}{l}\text { Method of Serum } \\
\text { 25(OH)D Measurement }\end{array}$ & Notable Findings \\
\hline $\begin{array}{l}\text { Unal et al., } \\
2017 \text { [40] }\end{array}$ & $\begin{array}{l}\text { AA-20 paediatric } \\
\text { patients } \\
\text { C-34 paediatric healthy } \\
\text { controls } \\
\text { Mean age of: } \\
\text { AA M/F-12.4 } \pm 4.2 / 13.3 \\
\pm 4.4 \text { years } \\
\text { C-M/F } 16.6 \pm 0.8 / 16.5 \\
\pm 1.01 \text { years }\end{array}$ & $\begin{array}{l}\text { S1-6 patients } \\
\text { S2-9 patients } \\
\text { S3-5 patients }\end{array}$ & $\mathrm{D} \leq 20 \mathrm{ng} / \mathrm{mL}$ & NA & $\begin{array}{l}\text { Vitamin D deficiency in both } \\
\text { groups with no significant } \\
\text { differences between the groups } \\
(p=0.084) . \\
\text { Significant inverse correlation } \\
\text { between serum } 25(\mathrm{OH}) \mathrm{D} \text { levels and } \\
\text { SALT score, disease duration and } \\
\text { number of patches }(p<0.001 \text {, } \\
r=-0.831, p<0.001, r=-0.997, \\
p<0.001, r=-0.989 \text { respectively }) .\end{array}$ \\
\hline $\begin{array}{l}\text { Rasheed et al., } \\
2012 \text { [41] }\end{array}$ & $\begin{array}{l}\text { TE- } 42 \text { patients } \\
\text { FPHL-38 patients } \\
\text { C-40 healthy controls } \\
\text { Mean age of: } \\
\text { TE and FPHL-29.8 } \pm 9.3 \\
\text { years } \\
\text { C-30.8 } \pm 8.56 \text { years }\end{array}$ & $\begin{array}{l}\text { TE: } \\
\text { Mild-22 patients } \\
\text { Moderate-6 patients } \\
\text { Severe-14 patients } \\
\text { FPHL: } \\
\text { Mild-15 patients } \\
\text { Moderate-13 patients } \\
\text { Severe-10 patients }\end{array}$ & $\begin{array}{l}\mathrm{S}>75 \mathrm{nmol} / \mathrm{L} \\
\mathrm{I}-25-75 \mathrm{nmol} / \mathrm{L} \\
\mathrm{D}<25 \mathrm{nmol} / \mathrm{L}\end{array}$ & $\begin{array}{l}\text { Competitive enzyme } \\
\text { immunoassay }\end{array}$ & $\begin{array}{l}\text { Significantly lower serum } 25(\mathrm{OH}) \mathrm{D} \\
\text { levels in TE and FPHL vs. C } \\
(p<0.001) . \\
\text { The highest serum } 25(\mathrm{OH}) \mathrm{D} \text { levels } \\
\text { in mild vs. severe FPHL and TE } \\
(p=0.035, p=0.203 \text { respectively). }\end{array}$ \\
\hline $\begin{array}{l}\text { Banihashemi et } \\
\text { al., 2016 [42] }\end{array}$ & $\begin{array}{l}\text { FPHL }-45 \text { patients; } \\
\text { C-45 healthy controls } \\
\text { Mean age of: } \\
\text { FPHL- } 29.11 \pm 7.31 \text { years } \\
\text { C-28.82 } \pm 7.11 \text { years }\end{array}$ & $\begin{array}{l}\text { Ludwig I-28 patients } \\
\text { Ludwig II-2 patients } \\
\text { Ludwig III-2 patients }\end{array}$ & $\begin{array}{l}\mathrm{S}>30 \mathrm{ng} / \mathrm{mL} \\
\mathrm{I}-20-30 \mathrm{ng} / \mathrm{mL} \\
\mathrm{D}<20 \mathrm{ng} / \mathrm{mL}\end{array}$ & ELISA & $\begin{array}{l}\text { Lower serum } 25(\mathrm{OH}) \text { D levels in } \\
\text { FPHL vs. C }(p=0.04) \text {. } \\
\text { No significant correlation between } \\
\text { serum } 25(\mathrm{OH}) \mathrm{D} \text { levels and } \\
\text { duration or severity of FPHL } \\
(p=0.77, p=0.92 \text { respectively). }\end{array}$ \\
\hline $\begin{array}{l}\text { Moneib et al., } \\
2014 \text { [43] }\end{array}$ & $\begin{array}{l}\text { FPHL-60 patients } \\
\text { C-60 healthy controls } \\
\text { Mean age of: } \\
\text { FPHL-26.4 } \pm 4.51 \text { years } \\
\text { C }-25.85 \pm 4.49 \text { years }\end{array}$ & $\begin{array}{l}\text { Ludwig I-34 patients } \\
\text { Ludwig II-22 patients } \\
\text { Ludwig III-4 patients }\end{array}$ & $\begin{array}{l}\mathrm{S}>30 \mathrm{ng} / \mathrm{mL} \\
\mathrm{I}-21-29 \mathrm{ng} / \mathrm{mL} \\
\mathrm{D}<20 \mathrm{ng} / \mathrm{mL} \\
\mathrm{IN}>150 \mathrm{ng} / \mathrm{mL}\end{array}$ & RIA & $\begin{array}{l}\text { Significantly lower mean serum } \\
25(\mathrm{OH}) \mathrm{D} \text { level in FPHL vs. C } \\
(p=0.0001) \text {. } \\
\text { Significant difference between } \\
\text { serum 25(OH)D levels and } \\
\text { Ludwig's three degrees }(p=0.006) \text {. } \\
\text { The highest serum 25(OH)D levels } \\
\text { in Ludwig III. }\end{array}$ \\
\hline
\end{tabular}


Table 1. Cont.

\begin{tabular}{|c|c|c|c|c|c|}
\hline \multirow[b]{2}{*}{ Authors } & \multirow{2}{*}{$\begin{array}{l}\text { Study Subjects (Number } \\
\text { and Age) }\end{array}$} & \multirow{2}{*}{ Severity of Alopecia } & \multicolumn{3}{|c|}{ Serum Concentration of Vitamin D $(25(\mathrm{OH}) \mathrm{D})$} \\
\hline & & & $\begin{array}{l}\text { Criteria for Vitamin D } \\
\text { Status }\end{array}$ & $\begin{array}{l}\text { Method of Serum } \\
\text { 25(OH)D Measurement }\end{array}$ & Notable Findings \\
\hline $\begin{array}{l}\text { Nayak et al., } \\
2016[44]\end{array}$ & $\begin{array}{l}\text { Diffuse hair loss-22 } \\
\text { patients } \\
\mathrm{C}-22 \text { healthy controls } \\
\text { Mean age of the study } \\
\text { population-20.89 years }\end{array}$ & NA & $\begin{array}{l}\mathrm{I}-25-75 \mathrm{nmol} / \mathrm{L} \\
\mathrm{D}<20-25 \mathrm{nmol} / \mathrm{L}\end{array}$ & ELISA & $\begin{array}{l}\text { Significantly lower serum } 25(\mathrm{OH}) \mathrm{D} \\
\text { levels among cases vs. C ( } p=0.007) \text {. }\end{array}$ \\
\hline $\begin{array}{l}\text { Karadag et al., } \\
2011 \text { [45] }\end{array}$ & $\begin{array}{l}\text { TE-63 patients } \\
\text { C-50 healthy controls } \\
\text { Mean age of: } \\
\text { TE-29.0 } \pm 11.9 \text { years } \\
\text { C }-28.4 \pm 9.4 \text { years }\end{array}$ & $\begin{array}{l}\text { Acute TE-29 patients } \\
\text { Chronic TE-34 patients }\end{array}$ & NA & RIA & $\begin{array}{l}\text { Significantly higher serum } \\
25(\mathrm{OH}) \mathrm{D} \text { levels in TE patients vs. C } \\
(p<0.01) \text {. } \\
\text { Significantly increased risk for TE } \\
\text { for patients with } 25(\mathrm{OH}) \mathrm{D} \text { levels in } \\
\text { the highest quadrant vs. the lowest } \\
\text { one }(p<0.0001) \text {. }\end{array}$ \\
\hline
\end{tabular}

AA-alopecia areata; AT—alopecia totalis; AU—alopecia universalis; TE—telogen effluvium; FPHL—female pattern hair loss; V—vitiligo; C—healthy controls; NA—not applicable; D—vitamin D deficiency; I-vitamin D insufficiency; S-vitamin D sufficiency; IN—vitamin D intoxication; S1—<25\% scalp hair loss; S2—25-49\% scalp hair loss; S2*-26-50\% scalp hair loss; S3-50-74\% scalp hair loss; S4-75-99\% scalp hair loss; S5-100\% scalp hair loss; SALT—Severity of Alopecia Tool; F-female; M-male; PTH-parathyroid hormone; 25(OH)D—25-hydroxyvitamin D; 1,25(OH $)_{2} \mathrm{D}-1,25$ dihydroxyvitamin D; CHL—chemilumiscence; EIA—enzyme immunoassay; ELISA—enzyme-linked immunosorbent assay; HPLC—high performance liquid chromatography; RIA—radioimmunoassay; LC-MS/MS—liquid chromatography/tandem mass spectrometry. Factor to convert units of 25(OH)D from $\mathrm{ng} / \mathrm{mL}$ into $\mathrm{nmol} / \mathrm{L}: 1 \mathrm{ng} / \mathrm{mL}=0.400641 \mathrm{nmol} / \mathrm{L}[46]$. 
Since the majority of studies were conducted on adult patients, the study by Unal et al., is of special interest. The authors demonstrated vitamin D deficiency in paediatric patients with AA and in the control group. However, they reported significant inverse correlation between serum $25(\mathrm{OH}) \mathrm{D}$ levels and disease severity, duration, as well as the number of bald patches [40]. The authors suggested that vitamin D deficiency may aggravate the disease and lead to severe hair loss [40].

The potential role of VDR in the pathogenesis of AA was also assessed. Fawzi et al. revealed significantly lower levels of tissue and serum VDR in AA than in a control group. An important negative correlation was observed between the extent of AA and tissue VDR [47]. The results are in accordance with the study by Lim et al. [48]. The authors found significantly lower expression of VDR in hair follicles and epidermis within alopecia lesions than in healthy skin. Moreover, the VDR levels were lower in patients with a more severe form of hair loss. Reduction of VDR expression in AA was related to decreased hair cycle-related signals-Wnt/ $\beta$-catenin signals. The authors suggested that reduced expression of VDR in AA might be related primarily to suppression of cell differentiation, since decreased expression of involucrin and filaggrin within hair follicles and epidermis were revealed [48].

Despite the fact that many studies reported deficiency or insufficiency of vitamin D in patients with AA, few reports evaluate the topical application of vitamin D analogues as a therapeutic option. Therefore, data reported by Kim et al., appear interesting [19]. The authors observed complete hair regrowth in the affected area after application of a topical calcipotriol solution $(50 \mu \mathrm{g} / \mathrm{mL})$ for 3 months in a 7-year old boy. During a 6-month follow-up, no hair loss was observed. A skin biopsy performed before treatment revealed loss of VDR expression in affected hair follicles. VDR expression was detected again after hair regrowth [19]. The results of these studies seem to confirm the correlation between expression of VDR and clinical hair regrowth, and highlight the role of vitamin D in AA.

Later, the effectiveness of topical application of $0.005 \%$ calcipotriol was evaluated in 22 adult patients with AA and scalp involvement lower than $40 \%$. After three months of therapy, $59.1 \%$ of patients had hair regrowth; no response was observed in $36.4 \%$ of patients and worsening in $0.04 \%$. A better clinical effect was observed in patients with a single bald patch. Among all patients, $91 \%$ had vitamin D deficiency. Interestingly, faster hair regrowth was observed in patients with lower serum 25(OH)D levels [49]. Similarly, improvement of mild-moderate AA after topical application of calcipotriol cream was also reported [50].

To sum up, the observed decreased serum 25(OH)D levels in AA patients suggest its role in the disease pathogenesis. It was demonstrated that vitamin D shifts the Th1 immune response toward Th2. Since AA is mainly Th1-mediated, the vitamin D deficiency may be involved in the development of hair loss [31,35]. The main limitation of the studies are small study groups, different inclusion criteria, and collection of samples during different seasons.

\section{Vitamin D in Female Pattern Hair Loss}

Female pattern hair loss (FPHL) is one of the most common types of alopecia in women. Clinically, it is characterized by diffuse hair shedding with maintained frontal hairline [51]. Recent literature data include genetic, hormonal, and environmental factors in the pathogenesis of FPHL [52]. The possible link between serum 25-hydroxyvitamin D and FPHL has been suggested since its decreased concentration was demonstrated in patients with FPHL compared to control group [41-43] (Table 1).

Moneib et al. reported a significantly lower serum 25(OH)D levels in patients with FPHL than in controls [43]. The majority of patients with FPHL (96.6\%) showed a vitamin D deficiency or insufficiency. Sufficient levels were observed only in 3.3\% of patients. There was no significant difference between different serum $25(\mathrm{OH}) \mathrm{D}$ levels and mean disease duration or patients' age; however, a significant difference between the severity of hair loss and mean serum $25(\mathrm{OH}) \mathrm{D}$ concentration was observed. The authors suggested that the higher serum $25(\mathrm{OH}) \mathrm{D}$ concentration in patients with most severe hair loss in comparison with less severe alopecia may result from increased exposure to ultraviolet light due to more decreased scalp hair density [43]. Contrary to this, in another study, patients with mild and moderate FPHL had significantly higher mean serum levels of $25(\mathrm{OH}) \mathrm{D}$ 
compared to those suffering from the severe form [41]. It cannot be excluded that conflicting results observed in both studies were determined by different patterns of sun exposure and evaluation of serum $25(\mathrm{OH}) \mathrm{D}$ level in different parts of the year.

It has also been pointed out that women with positive family history of FPHL and vitamin D deficiency or insufficiency are more prone to develop FPHL in comparison with women with sufficient serum 25(OH)D levels [43]. Contrary to this, Banihashemi et al. did not find any significant correlation between serum 25(OH)D concentration and positive family history of FPHL [42].

Although a decreased serum 25(OH)D concentration was demonstrated in FPHL, the role of VDR in FPHL was assessed in a single study. Fawzi et al. [47] reported significantly decreased concentrations of both serum and tissue VDR in androgenic alopecia in comparison with healthy controls. No correlation was observed between serum or tissue VDR concentration and disease severity. Interestingly, female patients had higher levels of both serum and tissue VDR than male patients [47]. This could be explained by the interaction of $17 \beta$-estradiol and $1,25(\mathrm{OH})_{2} \mathrm{D}$, which results in enhancement of $V D R$ gene expression [53].

At present, there are no studies evaluating the oral supplementation of vitamin D or its topical application in patients with FPHL.

The results described above suggest a possible involvement of vitamin D in pathogenesis of FPHL. However, the studies were limited by the small number of enrolled patients and different inclusion criteria. To elucidate the role of vitamin D in FPHL, further large-scale studies are required [41].

\section{Vitamin D in Telogen Effluvium}

Telogen effluvium (TE) is a common clinical problem defined as a non-scarring, diffuse hair loss that usually occurs 3 months after exposure to a triggering factor and is usually self-limiting. Causative factors considered in the pathogenesis include: stress, febrile states, drugs, endocrine abnormalities, and nutritional disturbances [54].

In a study conducted by Rasheed et al., the authors compared serum $25(\mathrm{OH}) \mathrm{D}$ levels in female patients with chronic TE, FPHL, and healthy controls. They revealed significantly lower serum $25(\mathrm{OH}) \mathrm{D}$ levels in comparison to the control group. The lowest level was observed in patients with the most severe hair loss. According to the authors, the results may indicate that vitamin D is involved in TE [41]. Similar results were reported by Nayak et al. [44]. Interestingly, the levels were significantly lower in female patients when compared with the females in the control group, whereas the difference between serum 25(OH)D concentrations in male cases and controls was not significant [44]. Contrary to these studies, Karadag et al. found significantly higher serum 25(OH)D levels in patients with TE than in the control group [45]. Moreover, serum 25(OH)D concentration in the higher quadrant was associated with a higher risk of developing TE. According to the authors, the observed increased serum 25(OH)D levels in TE might not be the cause, but rather a compensatory effect to the hair loss [45]. Loss of melanin synthesis in telogen hair follicles may result in increased vitamin D synthesis in the skin [55]. It was suggested that screening for vitamin D might be beneficial in the management of TE; however, data on the effects of vitamin D supplementation in hair loss is lacking.

Due to the limited number of studies and different methodologies it is difficult to compare the results. However, since nutritional disturbances are one of the triggering factors causing hair loss, the possible role of vitamin D or its deficiency should be studied further.

\section{Conclusions}

The studies revealed important alterations in serum 25(OH)D levels in patients with different types of non-scarring alopecia. In the majority of reports, the mean serum levels of $25(\mathrm{OH}) \mathrm{D}$ were decreased when compared to the healthy control, which might suggest a causal role of this decrease in the pathogenesis of hair loss. However it cannot be excluded that observed low serum $25(\mathrm{OH}) \mathrm{D}$ levels are secondary to the disease. Emotional stress related to severe hair loss might discourage patients form going outdoors as much as those without this condition. Therefore their potential 
for vitamin D synthesis might be reduced. Despite different mechanisms of AA, FPHL, and TE, it seems a reasonable therapeutic strategy to monitor serum $25(\mathrm{OH}) \mathrm{D}$ levels and introduce vitamin $\mathrm{D}$ supplementation in case of its deficiency or insufficiency in patients with AA, TE, and FPHL. It is worth noting that due to different methods used to evaluate the serum concentration of $25(\mathrm{OH}) \mathrm{D}$, caution should be warranted when comparing the results. Other limitations of the studies include unequal study groups, different inclusion criteria, and the possible influence of seasonal variability of serum $25(\mathrm{OH}) \mathrm{D}$ concentration, since the samples were collected during different seasons. Therefore, there is an urgent need to standardize the laboratory measurement of vitamin D status that would be accurate, comparable over time, localization, or laboratory [56,57]. Further studies including larger groups of patients with standardized inclusion criteria and standardized protocols of 25(OH)D measurement are required to evaluate the vitamin D status and the effect of vitamin D supplementation in alopecia.

Acknowledgments: The publication fee was covered by DS 164 of the Medical University of Lublin.

Author Contributions: Agnieszka Gerkowicz performed literature research and wrote the manuscript; Katarzyna Chyl-Surdacka performed literature research and contributed to manuscript editing; Dorota Krasowska carefully revised the manuscript for important intellectual content, Grażyna Chodorowska carefully revised the manuscript for important intellectual content and supervised the study. All authors read and approved the manuscript.

Conflicts of Interest: The authors declare no conflict of interest.

\section{References}

1. Mostafa, W.Z.; Hegazy, R.A. Vitamin D and the skin: Focus on a complex relationship: A review. J. Adv. Res. 2015, 6, 793-804. [CrossRef] [PubMed]

2. Veldurthy, V.; Wei, R.; Oz, L.; Dhawan, P.; Jeon, Y.H.; Christakos, S. Vitamin D, calcium homeostasis and aging. Bone Res. 2016, 4, 16041. [CrossRef] [PubMed]

3. Lips, P.; van Schoor, N.M. The effect of vitamin D on bone and osteoporosis. Best Pract. Res. Clin. Endocrinol. Metab. 2011, 25, 585-591. [CrossRef] [PubMed]

4. Lehman, B. Role of vitamin D3 pathway in healthy and diseased skin-Facts, contradictions and hypotheses. Exp. Dermatol. 2009, 18, 97-108. [CrossRef] [PubMed]

5. Rosen, Y.; Daich, J.; Soliman, I.; Brathwaite, E.; Shoenfeld, Y. Vitamin D and autoimmunity. Scand. J. Rheumatol. 2016, 45, 439-447. [CrossRef] [PubMed]

6. Xu, H.; Soruri, A.; Gieseler, R.K.; Peters, J.H. 1,25-Dihydroxyvitamin D3 exerts opposing effects to IL-4 on MHC class-II antigen expression, accessory activity, and phagocytosis of human monocytes. Scand. J. Immunol. 1993, 38, 535-540. [CrossRef] [PubMed]

7. Wang, T.T.; Nestel, F.P.; Bourdeau, V.; Nagai, Y.; Wang, Q.; Liao, J.; Tavera-Mendoza, L.; Lin, R.; Hanrahan, J.W.; Mader, S.; et al. Cutting Edge: 1,25-Dihydroxyvitamin D3 Is a Direct Inducer of Antimicrobial Peptide Gene Expression. J. Immunol. 2004, 173, 2909-2912. [CrossRef] [PubMed]

8. Penna, G.; Adorini, L. 1 $\alpha, 25$-Dihydroxyvitamin D3 inhibits differentiation, maturation, activation, and survival of dendritic cells leading to impaired alloreactive T cell activation. J. Immunol. 2000, 164, 2405-2411. [CrossRef] [PubMed]

9. Hewison, M. An update on vitamin D and human immunity. Clin. Endocrinol. 2012, 76, 315-325. [CrossRef] [PubMed]

10. Chang, S.H.; Chung, Y.; Dong, C.J. Vitamin D suppresses Th17 cytokine production by inducing C/EBP homologous protein (CHOP) expression. Biol. Chem. 2010, 285, 38751-38755. [CrossRef] [PubMed]

11. Chen, S.; Sims, G.P.; Chen, X.X.; Gu, Y.Y.; Chen, S.; Lipsky, P.E. Modulatory effects of 1,25-dihydroxyvitamin D3 on human B cell differentiation. J. Immunol. 2007, 179, 1634-1647. [CrossRef] [PubMed]

12. Aksu Cerman, A.; Solak Sarikaya, S.; Altunay Kivanc, I. Vitamin D deficiency in alopecia areata. Br. J. Dermatol. 2014, 170, 1299-1304. [CrossRef] [PubMed]

13. D'Ovidio, R.; Vessio, M.; d'Ovidio, F.D. Reduced level of 25-hydroxyvitamin D in chronic/relapsing alopecia areata. Dermatoendocrinol 2013, 5, 271-273. [CrossRef] [PubMed]

14. Arnson, Y.; Amital, H.; Shoenfeld, Y. Vitamin D and autoimmunity: New aetiological and therapeutic considerations. Ann. Rheum. Dis. 2007, 66, 1137-1142. [CrossRef] [PubMed] 
15. Baeke, F.; Van Etten, E.; Gysemans, C.; Overbergh, L.; Chantal, M. Vitamin D signaling in immune-mediated disorders: Evolving insights and therapeutic opportunities. Mol. Asp. Med. 2008, 29, 376-387. [CrossRef] [PubMed]

16. Mahamid, M.; Abu-Elhija, O.; Samamra, M.; Mahamid, A.; Mahmud, N.W. Association between vitamin D levels and alopecia areata. Isr. Med. Assoc. J. 2014, 16, 367-370. [PubMed]

17. Bikle, D.D.; Oda, Y.; Tu, C.L.; Jiang, Y. Novel mechanisms for the vitamin D receptor (VDR) in the skin and in skin cancer. J. Steroid Biochem. Mol. Biol. 2015, 148, 47-51. [CrossRef] [PubMed]

18. Van Etten, E.; Mathieu, C. Immunoregulation by 1,25-dihydroxyvitamin D3: Basic concepts. J. Steroid Biochem. Mol. Biol. 2005, 97, 93-101. [CrossRef] [PubMed]

19. Kim, D.H.; Lee, J.W.; Kim, I.S.; Choi, S.Y.; Lim, Y.Y.; Kim, H.M.; Kim, B.J.; Kim, M.N. Successful treatment of alopecia areata with topical calcipotriol. Ann. Dermatol. 2012, 24, 341-344. [CrossRef] [PubMed]

20. Kuryłowicz, A.; Bednarczuk, T.; Nauman, J. The influence of vitamin D deficiency on cancers and autoimmune diseases development. J. Investig. Dermatol. 2002, 118, 11-16.

21. Malloy, P.J.; Feldman, D. The role of vitamin D receptor mutations in the development of alopecia. Mol. Cell. Endocrinol. 2011, 347, 90-96. [CrossRef] [PubMed]

22. Demay, M.B.; MacDonald, P.N.; Skorija, K.; Dowd, D.R.; Cianferotti, L.; Cox, M. Role of the vitamin D receptor in hair follicle biology. J. Steroid Biochem. Mol. Biol. 2007, 103, 344-346. [CrossRef] [PubMed]

23. Amor, K.T.; Rashid, R.M.; Mirmirani, P. Does D matter? The role of vitamin D in hair disorders and hair follicle cycling. Dermatol. Online J. 2010, 16. [CrossRef]

24. Shafeghati, Y.; Momenin, N.; Esfahani, T.; Reyniers, E.; Wuyts, W. Vitamin D-dependent rickets type II: Report of a novel mutation in the vitamin D receptor gene. Arch. Iran. Med. 2008, 11, 330-334. [PubMed]

25. Bergman, R.; Schein-Goldshmid, R.; Hochberg, Z.; Ben-Izhak, O.; Sprecher, E. The alopecias associated with vitamin D-dependent rickets type IIA and with hairless gene mutations: A comparative clinical, histologic, and immunohistochemical study. Arch. Dermatol. 2005, 141, 343-351. [CrossRef] [PubMed]

26. Bikle, D.D.; Elalieh, H.; Chang, S.; Xie, Z.; Sundberg, J.P. Development and progression of alopecia in the vitamin D receptor null mouse. J Cell. Physiol. 2006, 207, 340-353. [CrossRef] [PubMed]

27. Miller, J.; Djabali, K.; Chen, T.; Liu, Y.; Ioffreda, M.; Lyle, S.; Christiano, A.M.; Holick, M.; Cotsarelis, G. Atrichia caused by mutations in the vitamin D receptor gene is a phenocopy of generalized atrichia caused by mutations in the hairless gene. J. Investig. Dermatol. 2001, 117, 612-617. [CrossRef] [PubMed]

28. Sakai, Y.; Kishimoto, J.; Demay, M.B. Metabolic and cellular analysis of alopecia in vitamin D receptor knockout mice. J. Clin. Investig. 2001, 107, 961-966. [CrossRef] [PubMed]

29. Mady, L.J.; Ajibade, D.V.; Hsaio, C.; Teichert, A.; Fong, C.; Wang, Y.; Christakos, S.; Bikle, D.D. The Transient Role for Calcium and Vitamin D during the Developmental Hair Follicle Cycle. J. Investig. Dermatol. 2016, 136, 1337-1345. [CrossRef] [PubMed]

30. Alkhalifah, A. Alopecia areata update. Dermatol. Clin. 2013, 31, 93-108. [CrossRef] [PubMed]

31. Alkhalifah, A.; Alsantali, A.; Wang, E.; McElwee, K.J.; Shapiro, J. Alopecia areata update: Part I. Clinical picture, histopathology, and pathogenesis. J. Am. Acad. Dermatol. 2010, 62, 177-188. [CrossRef] [PubMed]

32. Jabbari, A.; Petukhova, L.; Cabral, R.; Clynes, R.; Christiano, A. Genetic basis of alopecia areata. A road map for translational research. Dermatol. Clin. 2013, 31, 109-117. [CrossRef] [PubMed]

33. Yilmaz, N.; Serarslan, G.; Gokce, C. Vitamin D concentrations are decreased in patients with alopecia areata. Vitam. Miner. 2012, 1, 105-109. [CrossRef]

34. Bakry, O.A.; El Farargy, S.M.; El Shafiee, M.K.; Soliman, A. Serum Vitamin D in patients with alopecia areata. Indian Dermatol. Online J. 2016, 7, 371-377. [CrossRef] [PubMed]

35. Ghafoor, R.; Anwar, M.I. Vitamin D Deficiency in Alopecia Areata. Br. J. Dermatol. 2017, 27, $200-202$.

36. Darwish, N.M.M.; Marzok, H.F.; Gaballah, M.A.M.; Adbellatif, H.E. Serum level of vitamin D in patients with alopecia areata. Egypt. J. Basic Appl. Sci. 2017, 4, 9-14. [CrossRef]

37. Attawa, E.M.; Kandil, A.H.; Elbalaat, W.; Samy, A.M. Assessment of Vitamin D Level in Patients of Alopecia Areata. J. Clin. Investig. Dermatol. 2016, 4, 4.

38. Erpolat, S.; Sarifakioglu, E.; Ayyildiz, A. 25-hydroxyvitamin D status in patients with alopecia areata. Postepy Dermatol. Alergol. 2017, 34, 248-252. [CrossRef] [PubMed]

39. Bhat, Y.J.; Latif, I.; Malik, R.; Hassan, I.; Sheikh, G.; Lone, K.S.; Majeed, S.; Sajad, P. Vitamin D Level in Alopecia Areata. Indian J. Dermatol. 2017, 62, 407-410. [CrossRef] [PubMed] 
40. Unal, M.; Gonulalan, G. Serum vitamin D level is related to disease severity in pediatric alopecia areata. J. Cosmet. Dermatol. 2017. [CrossRef] [PubMed]

41. Rasheed, H.; Mahgoub, D.; Hegazy, R.; El-Komy, M.; Abdel Hay, R.; Hamid, M.A.; Hamdy, E. Serum ferritin and vitamin d in female hair loss: Do they play a role? Skin Pharmacol. Physiol. 2013, 26, 101-107. [CrossRef] [PubMed]

42. Banihashemi, M.; Nahidi, Y.; Meibodi, N.T.; Jarahi, L.; Dolatkhah, M. Serum Vitamin D3 Level in Patients with Female Pattern Hair Loss. Int. J. Trichol. 2016, 8, 116-120.

43. Moneib, H.; Fathy, G.; Ouda, A. Possible association of female-pattern hair loss with alteration in serum 25-hydroxyvitamin D levels. Egypt. J. Dermatol. Venerol. 2014, 34, 15-20.

44. Nayak, K.; Garg, A.; Mithra, P.; Manjrekar, P. Serum Vitamin D3 Levels and Diffuse Hair Fall among the Student Population in South India: A Case-Control Study. Int. J. Trichol. 2016, 8, 160-164. [CrossRef] [PubMed]

45. Karadag, A.S.; Ertugrul, D.T.; Tutal, E.; Akin, K.O. The role of anemia and Vitamin D levels in acute and chronic telogen effluvium. Turk. J. Med. Sci. 2011, 41, 827-833.

46. Endemo-Online Converters. Available online: http://www.endmemo.com/medical/unitconvert/Vitamin _D.php (accessed on 22 November 2017).

47. Fawzi, M.M.; Mahmoud, S.B.; Ahmed, S.F.; Shaker, O.G. Assessment of vitamin D receptors in alopecia areata and androgenetic alopecia. J. Cosmet. Dermatol. 2016, 15, 318-323. [CrossRef] [PubMed]

48. Lim, Y.Y.; Kim, S.Y.; Kim, H.M.; Li, K.S.; Kim, M.N.; Park, K.C.; Kim, B.J. Potential relationship between the canonical Wnt signalling pathway and expression of the vitamin D receptor in alopecia. Clin. Exp. Dermatol. 2014, 39, 368-375. [CrossRef] [PubMed]

49. Narang, T.; Daroach, M.; Kumaran, M.S. Efficacy and safety of topical calcipotriol in management of alopecia areata: A pilot study. Dermatol. Ther. 2017, 30, 3. [CrossRef] [PubMed]

50. Çerman, A.A.; Solak, S.S.; Altunay, İ.; Küçükünal, N.A. Topical Calcipotriol Therapy for Mild-to-Moderate Alopecia Areata: A Retrospective Study. J. Drugs Dermatol. 2015, 14, 616-620. [PubMed]

51. Olsen, E.A. Female pattern hair loss. J. Am. Acad. Dermatol. 2001, 45, S70-S80. [CrossRef] [PubMed]

52. Ramos, P.M.; Miot, H.A. Female Pattern Hair Loss: A clinical and pathophysiological review. An. Bras. Dermatol. 2015, 90, 529-543. [CrossRef] [PubMed]

53. Nashold, F.E.; Spach, K.M.; Spanier, J.A.; Hayes, C.E. Estrogen controls vitamin D3-mediated resistance to experimental autoimmune encephalomyelitis by controlling vitamin D3 metabolism and receptor expression. J. Immunol. 2009, 183, 3672-3678. [CrossRef] [PubMed]

54. Malkud, S. Telogen Effluvium: A Review. J. Clin. Diagn. Res. 2015, 9. [CrossRef] [PubMed]

55. Slominski, A.; Paus, R.; Costantino, R. Differential expression and activity of melanogenesis-related proteins during induced hair growth in mice. J. Investig. Dermatol. 1991, 96, 172-179. [CrossRef] [PubMed]

56. Sempos, C.T.; Durazo-Arvizu, R.A.; Binkley, N.; Jones, J.; Merkel, J.M.; Carter, G.D. Developing vitamin $\mathrm{D}$ dietary guidelines and the lack of 25-hydroxyvitamin $\mathrm{D}$ assay standardization: The ever-present past. J. Steroid Biochem. Mol. Biol. 2016, 164, 115-119. [CrossRef] [PubMed]

57. Cashman, K.D.; Kiely, M.; Kinsella, M.; Durazo-Arvizu, R.A.; Tian, L.; Zhang, Y.; Lucey, A.; Flynn, A.; Gibney, M.J.; Vesper, H.W.; et al. Evaluation of Vitamin D Standardization Program protocols for standardizing serum 25-hydroxyvitamin D data: A case study of the program's potential for national nutrition and health surveys. Am. J. Clin. Nutr. 2013, 97, 1235-1242. [CrossRef] [PubMed]

(C) 2017 by the authors. Licensee MDPI, Basel, Switzerland. This article is an open access article distributed under the terms and conditions of the Creative Commons Attribution (CC BY) license (http://creativecommons.org/licenses/by/4.0/). 